\title{
Point-of-Care Clinical Ultrasound for Medical Students
}

Authors

Affiliations
J. Heiberg ${ }^{1}$, L. S. Hansen ${ }^{2}$, K. Wemmelund ${ }^{2}$, A. H. Sørensen ${ }^{2}$, C. Ilkjaer ${ }^{1}$, E. Cloete ${ }^{3}$, D. Nolte ${ }^{3}$, F. Roodt ${ }^{3}$, R. Dyer ${ }^{3}$, J. Swanevelder ${ }^{3}$, E. Sloth

Affiliation addresses are listed at the end of the article
Key words

- abdomen

- heart

- vascular

ultrasound received 20.07.2015

accepted 23.10.2015

Bibliography

DOI http://dx.doi.org/

10.1055/s-0035-1565173

Published online:

November 6, 2015

Ultrasound International Open

2015; 1: E58-E66

(c) Georg Thieme Verlag KG

Stuttgart - New York

ISSN 2199-7152

Correspondence

\section{Dr. Johan Heiberg}

Department of Cardiothoracic

\& Vascular Surgery

Aarhus University Hospital

Palle Juul-Jensens Boulevard 99

Aarhus

Denmark 8200

Tel.: + 45/784/53 083

johan.heiberg@clin.au.dk

\section{License terms}

\section{Abstract}

$\nabla$

Purpose: Our institution has recently implemented a point-of-care (POC) ultrasound training program, consisting of an e-learning course and systematic practical hands-on training. The aim of this prospective study was to evaluate the learning outcome of this curriculum.

Materials and Methods: 16 medical students with no previous ultrasound experience comprised the study group. The program covered a combination of 4 well-described point-of-care (POC) ultrasound protocols (focus assessed transthoracic echocardiography, focused assessment with sonography in trauma, lung ultrasound, and dynamic needle tip positioning for ultrasoundguided vascular access) and it consisted of an e-learning course followed by $4 \mathrm{~h}$ of practical

\section{Introduction}

During the past decade, point-of-care (POC) ultrasound has been rapidly evolving and the topic constitutes a steadily growing number of publications [1-5]. Previous studies have focused predominantly on feasibility, but newer studies based on randomized, blinded and controlled designs have pointed out significant advantages both as a potential life-saving diagnostic tool [6$8]$, and as a guiding instrument in daily clinical procedures $[9,10]$. However, implementation into daily clinical practice is challenging and requires efficient educational programs covering both theory and practical skills $[11,12]$.

Medical students have been proven to learn quickly and may benefit from the skills achieved during their entire professional careers [13]. Several medical schools have integrated ultrasound as part of their teaching programs [14-16], but mainly as a learning tool in anatomy, physiology, and pathology [17-19]. The ultimate goal is to hands-on training. Practical skills and image quality were tested 3 times during the study: at baseline, after e-learning, and after hands-on training.

Results: Practical skills improved for all 4 protocols; after e-learning as well as after handson training. The number of students who were able to perform at least one interpretable image of the heart increased from 7 at baseline to 12 after e-learning, $\mathrm{p}<0.01$, and to all 16 students after hands-on-training, $\mathrm{p}<0.01$. The number of students able to cannulate an artificial vessel increased from 3 to 8 after e-learning and to 15 after hands-on training.

Conclusion: Medical students with no previous ultrasound experience demonstrated a considerable improvement in practical skill after interactive e-learning and $4 \mathrm{~h}$ of hands-on training.

benefit the patients by increased use of POC ultrasound and therefore, a foundation in POC ultrasound should be taught early in medical education [1,11,20-22].

In 2011, a major revision of the medical school curriculum at $\mathrm{X}, \mathrm{X}$, gave ultrasound a far greater prominence. As part of this transformation, all medical students were offered an introduction to POC ultrasound; a program consisting exclusively of e-learning-based theory and a 1-day hands-on training session. Although the feedback has been very positive, the optimal educational program for the learning of ultrasound remains debatable. Thus, our objective was to assess the learning outcome from our ultrasound curriculum, with particular focus on differentiating the value of theoretical e-learning and practical hands-on training on medical students with no prior experience in ultrasound. 


\section{Methods}

$\nabla$

We conducted a single center, prospective trial to test an interactive e-learning program followed by $4 \mathrm{~h}$ of ultrasound hands-on training. The timeline is displayed in $\bullet$ Fig. $\mathbf{1 .}$

\section{Study population}

20 young medical students with no previous ultrasound experience, all in their third to fifth year of medical school (mean $4.5 \pm 0.6$ ), at the University of X were voluntarily recruited. 4 students served as ultrasound models and 16 comprised the study group according to our preceding sample size calculation.

\section{Ultrasound curriculum}

Our ultrasound curriculum is entitled "Introduction to $\mathrm{ABC}$ Ultrasound", as it presents ultrasound as a diagnostic tool to evaluate the functions vital for life: Airway, Breathing, and Circulation. The program covers a combination of 4 POC protocols: 1) Focus Assessed Transthoracic Echocardiography (FATE) [23], 2) Focused Assessment with Sonography in Trauma (FAST) [24,25], 3) Lung Ultrasound (LUS) [22], and 4) Dynamic Needle Tip Positioning (DNTP) for ultrasound guided vascular access [26]. The program consists of 2 parts: an internet-based e-learning course and a systematic, structured hands-on training (HOT) session, as described below.

\section{E-learning}

The e-learning course consisted of interactive internet-based modules developed especially for the medical student curriculum. The modules included a combination of text, pictures/photos, animations, movies and tests to ensure maximal knowledge retention. Each of the modules had a logic progression in the subject and held information of how to acquire an image as well as how to interpret it. Typically, an instruction of how to acquire or interpret an image was given both as a text and as a video illustration. From previous experience, the e-learning required approximately $5-8 \mathrm{~h}$. As a part of the web-based e-learning program the students had their theoretical knowledge tested before and after the course with 56 multiple-choice questions, equally distributed among the 4 protocols. Students were requested to answer these questions without access to the internet or textbooks. Each of the 2 tests was done only once, and the response time and individual results of the students were automatically stored.

\section{HOT-session}

4 groups each consisting of 4 students, rotated between 4 HOTstations with an instructor and an ultrasound model at each station. Each group received $1.5 \mathrm{~h}$ of FATE-training, $1 \mathrm{~h}$ of FAST-training, $30 \mathrm{~min}$ of LUS-training, and $1 \mathrm{~h}$ of training in DNTP. The instructors were responsible for strict adherence to a time schedule, allowing each student the same time exposure to every station.

\section{Student evaluation}

3 test sessions were held: at baseline, after the e-learning course (intermediate test), and after the 4-h HOT session (endpoint test). All test sessions were performed identically, with the same instructors, models and students at each station. The 4 models were used only during the test sessions, and the students were not allowed to 'practice' on these individuals. In brief, 4 students were simultaneously tested at different test stations with rotations every $20 \mathrm{~min}$. 2 protocols were tested at each station with a total test time of $40 \mathrm{~min}$ per student. For each protocol, the tests comprised 2 types of evaluation: online testing of the students' practical skills by simple correct/incorrect questions, and offline evaluation of image quality in terms of adequacy of interpretation. For all 4 protocols the first practical exercise, before proceeding to the views, was to point out the most appropriate transducer. In case of an incorrect answer, the correct answer was given to make sure that the most appropriate transducer was used.

Prior to each test the instructor gave standardized information about the protocol tested:

A. Students were permitted $90 \mathrm{~s}$ for each view.

B. Students would receive help in storing and adjusting the image on request. Thereby, the students were responsible for determining when the image was an adequate representation of the expected image. Likewise, the image was only adjusted if the students requested a specific adjustment e.g., depth increase.

C. Up to 3 loops could be stored for each view.

D. Before each view the students had 30 s to examine a poster presented to them. While examining the poster the students were informed about the practical exercise related to the particular view, and that if he/she would not be able visualize the structures asked for, the exercise would be considered incorrect.

E. Time zero was the point at which the student first made contact with the volunteer. After $30 \mathrm{~s}$ they were reminded to store the images obtained, and after $60 \mathrm{~s}$ the instructor informed them that $30 \mathrm{~s}$ remained.

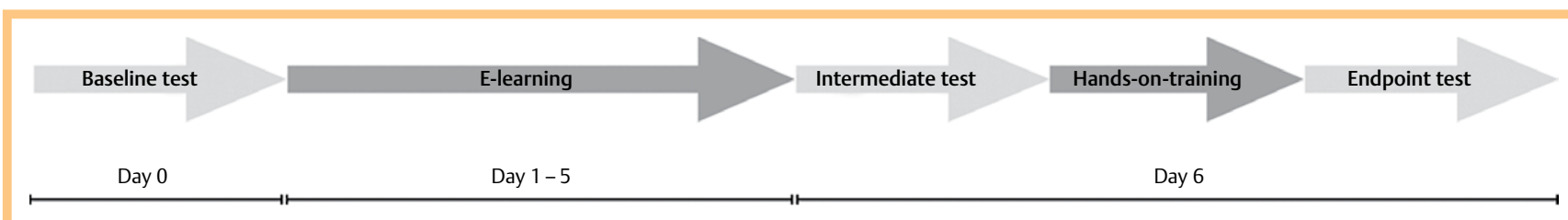

Fig. 1 Study timeline. Prior to the study, each student received a number from 1-16, and they were divided into groups of 4 (1-4, 5-8, 9-12, and 13-16). As displayed, they were tested 3 times during the study period: at baseline, after the e-learning course (intermediate test), and after the 4-h HOT session (endpoint test). Day 0: The students received an introduction of $15 \mathrm{~min}$, which included the practical arrangements and general information about the study, including the hypothesis and aim. No ultrasound theory was taught at this stage. Immediately after the introduction, the baseline test was conducted. Day 1-5: Electronic access codes to the e-learning course were distributed after all students had completed the baseline test. Day 6: After initial registration, no instructions were given prior to the intermediate test. Before the handson-training session, the students were briefly informed about the rotation scheme, but no ultrasound theory was taught. The endpoint test was carried out immediately after the hands-on training session. 
Models were only repositioned upon request, instructors answered no theoretical questions, and they responded similarly whether the exercises were solved correctly or not. The instructors were responsible for changing transducers between protocols, and keeping track of time.

1) The following cardiac and pleural views were tested Subcostal 4-chamber view, apical 4-chamber view, parasternal long-axis view, parasternal short-axis view, right pleural view, and left pleural view ( $\odot$ Table 1a and Appendix 1a).

\section{2) The following abdominal views were tested}

Subcostal 4-chamber view, right upper quadrant view, left upper quadrant view, and transverse pelvic view ( $\bullet$ Table 1b and Appendix 1b).

3) The following lung ultrasound features were tested "BAT-sign" in longitudinal view (across the ribs) and "lung sliding" in transverse view (intercostal space) ( $\bullet$ Table 1c and Appendix 1c).

\section{4) The following vascular views were tested}

Transverse views of peripheral vessels, artery and vein on the right forearm, and transverse view of the internal jugular vein. Tourniquets were only handed out when requested by the student ( $\bullet$ Table 1d and Appendix 1d).

\section{Offline analysis}

The stored images were evaluated in random order, and 2 blinded observers (JH, LSH) scored the best loops after consensus based on 10 full datasets. Scoring also required selection of the best loop. In case of divergence between the observers consensus was reached.

\section{1) Cardiac and pleural ultrasound}

An R-wave-triggered cine-loop was scored from 1-5 in terms of image quality, using a previously published algorithm [27] as follows: $1=$ no image, 2 =poor and unusable image quality, $3=$ usable image quality, $4=$ good image quality, and $5=$ perfect image quality. An image score $\geq 3$ was judged sufficient to assess dimensions, free fluid, and global function; in such cases the image could contribute to clinical decision-making.

In order to assess inter-observer variability, the 2 observers $(\mathrm{JH}$, LSH) scored all images from the 3 test sessions. Likewise, in order to assess intra-observer variability, one observer $(\mathrm{JH})$ rescored all 3 sets of cardiac and pleural images from 4 randomly selected students for each of the protocols. In total, the interand intra-observer variability tests compared 48 pairs and 12 pairs of observations, respectively.

\section{2-4) Abdominal, lung, and vascular ultrasound}

Loops of $3 \mathrm{~s}$ were scored on a 2-point scale, signifying whether the image was interpretable or not $(0=$ uninterpretable, $1=$ interpretable).

\section{Statistical analysis}

For statistical analyses and graphical description we used Stata/ IC 12.1 for Mac (StataCorp, TX, USA) and Graph Pad Prism 6 (GraphPad Software, La Jolla, CA, USA). Continuous, normally distributed data are reported as mean (standard deviation [SD]), otherwise as median and range, and the normally distributed data were compared applying one-way analysis of variance (ANOVA). If appropriate, paired Student's $t$-tests with either equal or unequal variance were used. The Wilcoxon rank sum test was applied for paired, continuous, non-normally distributed variables. Binominal data are presented as absolute numbers, and compared applying the chi-squared test. Correlations were checked applying simple linear regression analyses. We set a significance level of $95 \%$, but adjusted appropriately for multiple comparisons by the Bonferroni correction. Therefore, only $p$-values $<0.01$ were considered statistically significant.

Our preceding sample size calculation was based on the overall image score in cardiac and pleural ultrasound in which we hypothesized an increase of $20 \%$ from baseline test to endpoint test and standard deviations of $15 \%$ in both tests. With a statistical power of $90 \%$ and a level of significance of $95 \%$, we therefore needed 12 students to participate in the study.

\section{Ethics}

Prior to the study, The Human Research Ethics Committee of the Faculty of Health Sciences of the University of X approved the study. It was exempt from formal ethics approval in X, according to The Regional Committee on Biomedical Research Ethics of the $\mathrm{X}$. Each student signed an informed consent form agreeing to participate in the study.

\section{Results}

$\nabla$

The median e-learning score was $69(53-86 \%)$ in the pre e-learning test vs. $98(88-100 \%)$ in the post e-learning test, $p<0.01$. The time required for the pre e-learning test was $14: 56 \mathrm{~min}$ (9:56$25: 38)$ vs. $10: 43 \mathrm{~min}(5: 04-53: 18)$ for the post e-learning test, $p=0.08$. All the students completed the e-learning course, although 2 did not do the pre- and post e-learning test in succession, and their test time could therefore not be reliably assessed. The overall progress in test scores and image quality is shown in $\odot$ Fig. 2a, c.

\section{1) Cardiac and pleural ultrasound}

The number of correct answers increased from baseline to intermediate test in all the evaluated views except the ability to point out the right diaphragm ( $\bullet$ Table 1a). Also, the number of students able to perform an interpretable image increased in all the views, ( $\bullet$ Table 2a). We evaluated the ability to perform at least one interpretable image of the heart, and a significant increase from 7 at baseline to 12 at the intermediate test, $p<0.01$ was shown. The overall image score from the 6 views as percentage of the highest possible score rose from $38.3 \pm 11.0 \%$ of possible score at baseline to $54.0 \pm 14.0 \%$ at the intermediate test, $p<0.01$. From the intermediate test the students improved in all practical exercises except selection of transducers, which were chosen correctly by 15 out of 16 students at the endpoint test. All 16 students performed one or more interpretable images of the heart at the endpoint test with a significant increase from the intermediate test, $p<0.01$. The image score rose to $73.1 \pm 12.0 \%$ of highest possible score at the endpoint test, $p<0.01$, ( $\bullet$ Fig. $2 d$ ). In 2 students intermediate test scores were lower than baseline. One student had a lower endpoint score than intermediate score. In all students image scores improved from the baseline to the endpoint test. 
Table 1 Practical exercises in cardiac and pleural (a), abdominal (b), lung (c), and vascular ultrasound (d). Students with correct answers to each of the exercises.

\begin{tabular}{|c|c|c|c|c|}
\hline & Baseline-test $(\mathrm{N}=16)$ & Intermediate test $(\mathrm{N}=16)$ & Endpoint test $(\mathrm{N}=16)$ & $p$-value \\
\hline \multicolumn{5}{|l|}{ a - Cardiac and pleural ultrasound } \\
\hline \multicolumn{5}{|l|}{ Exercise } \\
\hline Selection of transducer? & 14 & 15 & 15 & 0.76 \\
\hline \multicolumn{5}{|l|}{ Point on the subxiphoid view: } \\
\hline LV (no.) & 2 & 12 & 14 & $<0.01^{*}$ \\
\hline RV (no.) & 3 & 12 & 15 & $<0.01^{*}$ \\
\hline LA (no.) & 2 & 10 & 13 & $<0.01^{*}$ \\
\hline RA (no.) & 2 & 8 & 13 & $<0.01^{*}$ \\
\hline \multicolumn{5}{|l|}{ Point on the apical 4-chamber view: } \\
\hline LV (no.) & 4 & 7 & 15 & $<0.01^{*}$ \\
\hline RV (no.) & 2 & 5 & 15 & $<0.01^{*}$ \\
\hline LA (no.) & 2 & 5 & 14 & $<0.01^{*}$ \\
\hline RA (no.) & 1 & 4 & 13 & $<0.01^{*}$ \\
\hline \multicolumn{5}{|l|}{ Point on the parasternal long-axis view: } \\
\hline LV (no.) & 1 & 10 & 15 & $<0.01^{*}$ \\
\hline RV (no.) & 2 & 9 & 14 & $<0.01^{*}$ \\
\hline LA (no.) & 1 & 8 & 14 & $<0.01^{*}$ \\
\hline Aortic outflow tract (no.) & 2 & 5 & 14 & $<0.01^{*}$ \\
\hline \multicolumn{5}{|l|}{ Point at the parasternal short-axis view: } \\
\hline LV (no.) & 5 & 14 & 16 & $<0.01^{*}$ \\
\hline RV (no.) & 4 & 11 & 14 & $<0.01^{*}$ \\
\hline \multicolumn{5}{|l|}{ Point on the left pleural view: } \\
\hline Diaphragm (no.) & 10 & 13 & 16 & 0.03 \\
\hline Liver (no.) & 13 & 14 & 16 & 0.21 \\
\hline \multicolumn{5}{|l|}{ Point on the right pleural view: } \\
\hline Diaphragm (no.) & 7 & 5 & 11 & 0.11 \\
\hline Spleen (no.) & 3 & 9 & 15 & $<0.01^{*}$ \\
\hline \multicolumn{5}{|l|}{ b - Abdominal ultrasound } \\
\hline \multicolumn{5}{|l|}{ Exercise } \\
\hline Selection of transducer? & 10 & 15 & 16 & $<0.01^{*}$ \\
\hline \multicolumn{5}{|l|}{ Point on the subxiphoid view: } \\
\hline LV (no.) & 4 & 7 & 11 & 0.05 \\
\hline RV (no.) & 4 & 7 & 11 & 0.05 \\
\hline LA (no.) & 3 & 6 & 10 & 0.04 \\
\hline RA (no.) & 3 & 7 & 12 & $<0.01^{*}$ \\
\hline \multicolumn{5}{|l|}{ Point on the left upper quadrant view: } \\
\hline Liver (no.) & 11 & 16 & 16 & $<0.01^{*}$ \\
\hline Kidney (no.) & 5 & 11 & 12 & 0.03 \\
\hline \multicolumn{5}{|l|}{ Point on the right upper quadrant view: } \\
\hline Spleen (no.) & 5 & 9 & 14 & $<0.01^{*}$ \\
\hline Kidney (no.) & 3 & 7 & 15 & $<0.01^{*}$ \\
\hline \multicolumn{5}{|l|}{ Point on the transverse pelvic view: } \\
\hline Bladder (no.) & 4 & 8 & 12 & 0.02 \\
\hline \multicolumn{5}{|l|}{ c - Lung ultrasound } \\
\hline Exercise & & & & \\
\hline Selection of transducer? & 12 & 12 & 15 & 0.29 \\
\hline Point on a transverse view: & & & & \\
\hline Costa shadow (no.) & 6 & 15 & 16 & $<0.01^{*}$ \\
\hline Pleura line (no.) & 7 & 15 & 16 & $<0.01^{*}$ \\
\hline Point on a longitudinel view: & & & & \\
\hline Pleura line (no.) & 8 & 16 & 16 & $<0.01^{*}$ \\
\hline Lung sliding (no.) & 1 & 13 & 15 & $<0.01^{*}$ \\
\hline d - Vascular ultrasound & & & & \\
\hline Exercise & & & & \\
\hline Selection of transducer? (no.) & 10 & 14 & 16 & 0.02 \\
\hline Perform finger test? (no.) & 3 & 8 & 16 & $<0.01^{*}$ \\
\hline Perform compression test? (no.) & 1 & 9 & 11 & $<0.01^{*}$ \\
\hline Point out the following vessels: & & & & \\
\hline Peripheral vein (no.) & 6 & 5 & 10 & 0.17 \\
\hline Int. jugular vein (no.) & 14 & 16 & 16 & 0.12 \\
\hline Carotid artery (no.) & 15 & 16 & 16 & 0.36 \\
\hline Vascular access in a phantom: * * & & & & \\
\hline Within 90 s? (no.) & 3 & 8 & 15 & $<0.01^{*}$ \\
\hline
\end{tabular}



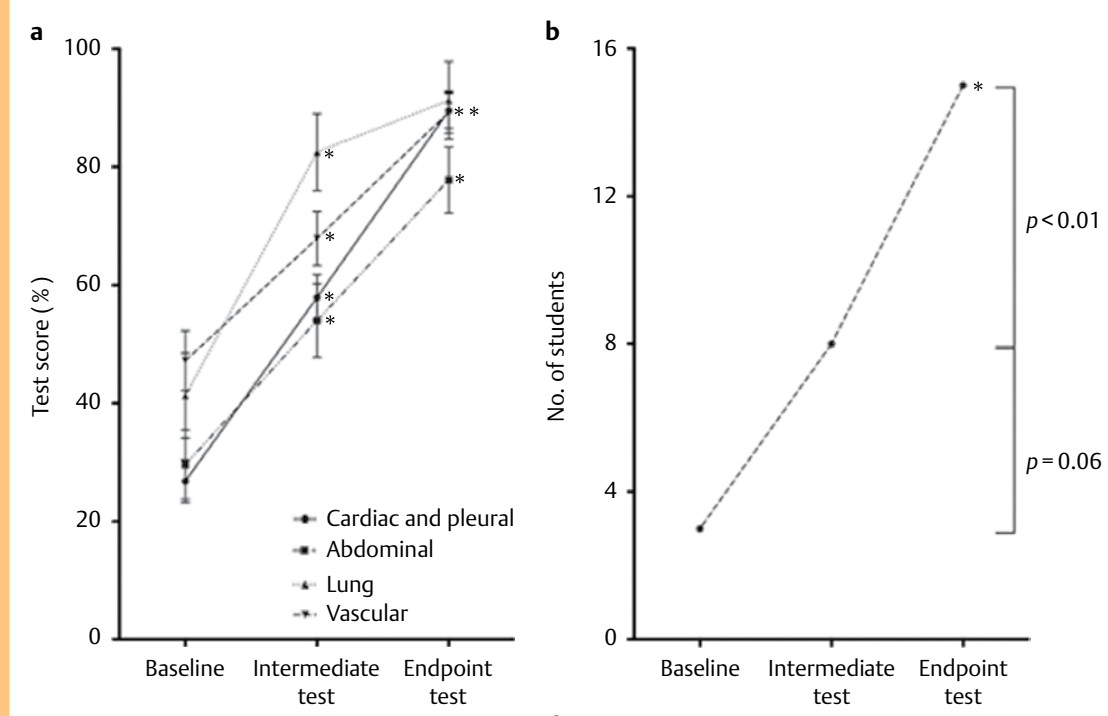

Fig. 2 Development in practical exercise scores and image quality. Online evaluation of practical skills $\mathbf{a}, \mathbf{b}$ and offline evaluation of image quality c, d. a Scores as percentage of highest possible score for each protocol, $p<0.001$ (ANOVA) for all 4 protocols. $\mathbf{b}$ Example of development in practical skills; vascular access performed within $90 \mathrm{~s}$, at the 3 tests, $p<0.001$ (ANOVA). $c$ Number of interpretable images as percentage of highest possible number for each protocol, $p<0.001$ (ANOVA) for FATE, FAST, and LUS; $\boldsymbol{d}$ Image score from FATE presented as percentage of the highest possible score, $p<0.001$ (ANOVA). * Significantly different from previous test, $p<0.001$. ANOVA: one-way analysis of variance.

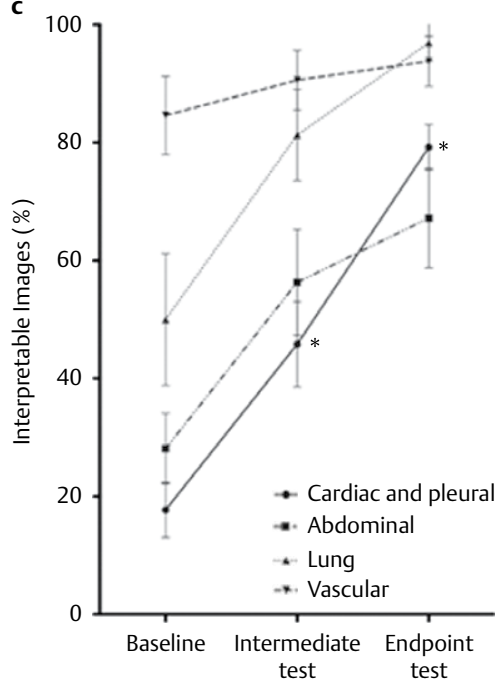

d

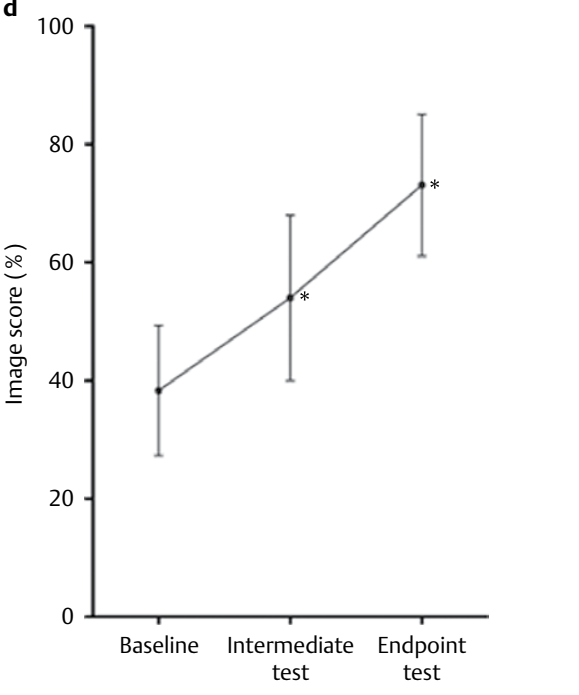

\section{2) Abdominal ultrasound}

From baseline to the intermediate test the number of correct answers in the practical exercises improved in all the questions ( $\odot$ Table 1b). Likewise, an increasing number of students performed interpretable images in all evaluated views ( $\boldsymbol{\Theta}$ Table $\mathbf{2 b}$ ). From intermediate test to endpoint test, a similar trend was seen with improvement in all exercises and image quality in all views. In 4 students, image scores were lower for the intermediate test than the baseline test, and in another 4 students performance was weaker in the endpoint test than the intermediate test. All students had higher image scores at the end of the study than at baseline.

\section{3) Lung ultrasound}

The students improved from baseline to intermediate test in all the exercises except transducer selection ( $\bullet$ Table 1c), and the number of interpretable views increased similarly ( $\bullet$ Table $\mathbf{2 c}$ ). At the endpoint test all 16 students were able to point out the pleura line and the rib shadow, likewise 15 out of 16 students answered the 2 remaining questions correctly at the final test. Offline image evaluation showed that all 16 students were able to perform the transverse view suitably for interpretation, and 15 of the students performed a clinically usable longitudinal view at the endpoint test.

\section{4) Vascular ultrasound}

There was an increase in the number of correct answers in all exercises from baseline to intermediate test ( $\bullet$ Table 1d). All 16 students were able to perform an interpretable image of the internal jugular vein as well as the carotid artery, and could also identify the structures correctly ( $\bullet$ Table 2 d). Within $90 \mathrm{~s}$, 3 students succeeded in vessel cannulation at baseline compared with 8 in the intermediate test. At the last test 15 performed a successful vascular access ( $\bullet$ Fig. 2b). Likewise, 15 students produced interpretable images at the endpoint test ( $\boldsymbol{O}$ Table $2 \mathbf{d}$ ).

\section{Discussion}

This study establishes that interactive e-learning in combination with $4 \mathrm{~h}$ of systematic hands-on training can provide considerable improvement in ultrasound competence among medical students with no previous ultrasound experience. Our data reflected the transition from absolute no training, wherefore rapid progression must be expected no matter the type of training. However, clearly the learning curves in practical skills after the theoretical e-learning course alone are remarkably steep. Today only few medical schools have POC ultrasound as an integrated part of their curricula [14-16]. Hoppmann et al. described 


\begin{tabular}{|c|c|c|c|c|}
\hline & $\begin{array}{l}\text { Baseline-test }{ }^{* *} \\
(\mathrm{~N}=16)\end{array}$ & $\begin{array}{l}\text { Intermediate test } \\
(\mathrm{N}=16)\end{array}$ & $\begin{array}{l}\text { Endpoint test } \\
(\mathrm{N}=16)\end{array}$ & $p$-value \\
\hline \multicolumn{5}{|l|}{ a - Cardiac and pleural } \\
\hline Subcostal 4-chamber view (no.) & 2 & 8 & 15 & $<0.01^{*}$ \\
\hline Apical 4-chamber view (no.) & 4 & 6 & 13 & $<0.01^{*}$ \\
\hline Parasternal long-axis view (no.) & 3 & 7 & 12 & $<0.01^{*}$ \\
\hline Parasternal short-axis view (no.) & 2 & 10 & 12 & $<0.01^{*}$ \\
\hline Right pleural view (no.) & 5 & 9 & 16 & $<0.01^{*}$ \\
\hline Left pleural view (no.) & 1 & 4 & 8 & 0.02 \\
\hline \multicolumn{5}{|l|}{ b - Abdominal } \\
\hline Subcostal 4-chamber view (no.) & 6 & 8 & 10 & 0.37 \\
\hline Right upper quadrant view (no.) & 7 & 12 & 13 & 0.06 \\
\hline Left upper quadrant view (no.) & 2 & 8 & 12 & $<0.01^{*}$ \\
\hline Transverse pelvic view (no.) & 3 & 8 & 8 & 0.11 \\
\hline \multicolumn{5}{|l|}{ c- Lung } \\
\hline BAT-sign (no.) & 8 & 12 & 16 & $<0.01^{*}$ \\
\hline Lung sliding (no.) & 8 & 14 & 15 & $<0.01^{*}$ \\
\hline \multicolumn{5}{|l|}{ d - Vascular } \\
\hline Peripheral vessels (no.) & 10 & 13 & 15 & 0.42 \\
\hline Central vessels (no.) & 12 & 16 & 15 & 0.55 \\
\hline
\end{tabular}

an ultrasound program implemented across all 4 years in medical school at the University of South Carolina [14], while others have reported on programs introduced during the last year of medical school $[15,16]$. These previous experiences all demonstrated how POC ultrasound could be successfully implemented in medical schools with high learning outputs and excellent student feedback. Other studies have evaluated various methods of POC ultrasound training programs for medical students; however, these were mainly non-implemented programs conducted on a pilot basis $[28,29]$. These investigations also concluded that ultrasound training should be included in the undergraduate medical school curriculum. Clearly, no course is complete without both a theoretical as well as a hands-on component, but the present study went beyond the above quoted studies in that the outcome was assessed after each separate element of the course, i. e., the theoretical and the practical training ( $\bullet$ Fig. 3 ).

In the current study we evaluated an ultrasound curriculum without 'classic' didactic lecturing, but instead with an internetbased e-learning course. E-learning offers substantial advantages. Firstly, the e-learning concept includes an increased accessibility to information, e.g., the students can access what is needed, when it is needed. Secondly, the electronic content is easy to update in comparison with printed material, and can quickly be revised or simplified. Thirdly, students are in control of their learning sequence, pace of learning, and study venue. Fourthly, e-learning standardizes the course content and delivery, in contrast to a lecture. Lastly, e-learning can be designed to include an outcome assessment to determine whether knowledge has been acquired. Although our study was not designed to compare e-learning-based theory with other teaching modalities, our data suggest that didactic lecturing can be replaced with an interactive e-course without compromising the acquisition of knowledge and understanding.

The majority of studies in the field are conducted employing time-consuming programs, whereas only a few studies have addressed learning outcomes after a shorter exposure, i.e., 1 day or less [28-30]. In the present study, a limited program of only $4 \mathrm{~h}$ of practical hands-on training was evaluated. $\odot$ Fig. 2 shows the rapid progress made. For example, 15 out of 16 students were able to place a needle in an artificial vessel at the end of the study. In our opinion, this strongly emphasizes the importance of an appropriate approach to the practical teaching, i.e., not spending excessive amounts of time showing the students a particular procedure, but allowing them to practice themselves from the beginning under the guidance of a supervisor. Moreover, it should be added that in the current study we restricted the HOT session to $4 \mathrm{~h}$, which was practically feasible and relatively easy to extend. In the ultrasound-training program implemented at Aarhus University, the HOT-session has a 6-h timeframe. Thus, the HOT session can easily be extended and still be completed within a 1-day time period. Such an extension may further improve the learning outcomes.

Danish medical students are all exposed to ultrasound techniques during their education. We therefore enrolled medical students from the University of $\mathrm{X}, \mathrm{X}$, who had no previous ultrasound experience. Our data therefore exclusively reflects the learning achieved by the interventions. Furthermore, the models used for the tests were only examined during these test sessions, and no 'practicing' was allowed on these individuals. Although the students might become more familiar with a particular model's anatomy from one test to another, the benefit of using the same models for all the test sessions, significantly outweighs this potential risk.

As previous suggested $[11,13]$, our data indicate that medical students are relatively quick learners in comparison with their older colleagues. In addition, we believe that clinicians are more likely to incorporate ultrasound into their daily practice if it is introduced at an early stage of their careers. Therefore, it is very important to integrate POC ultrasound into early medical education. The current study conducted on medical students at minimal cost and effort has shown significant improvement in their ability to obtain and interpret ultrasound images.

\section{Limitations}

$\nabla$

Firstly, we designed our study to focus more on the student's practical and technical ultrasound skills than on 'knobology', 

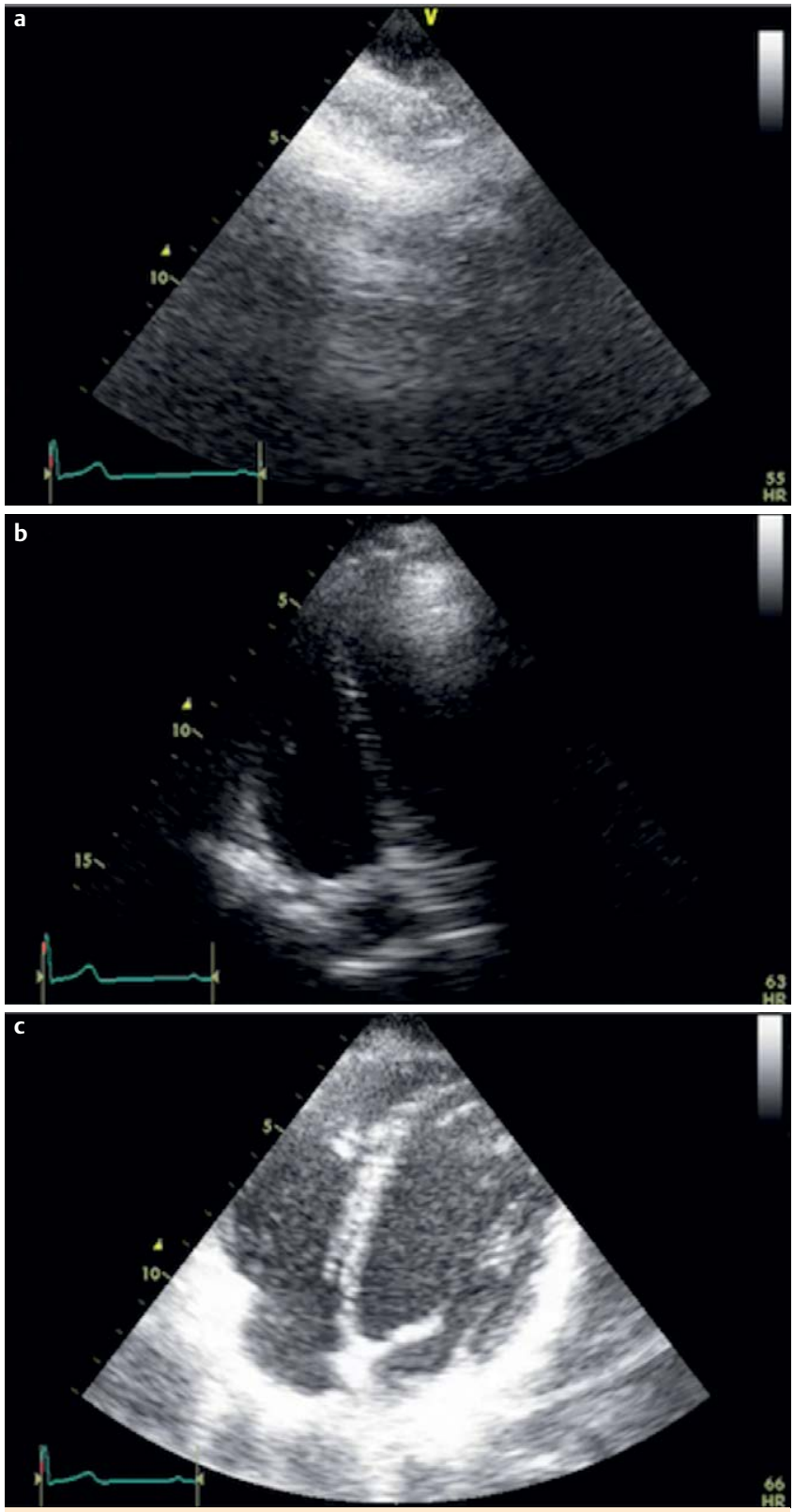

Fig. 3 Example of image quality development. Apical 4-chamber view from the 3 tests (student no. 15). a Baseline test; $\mathbf{b}$ Intermediate test; c Endpoint test.

and therefore, we chose to have the instructors assist with the storing of images if requested. Likewise, the instructors were responsible for changing transducers between protocols.

Secondly, we did not assess the students' ability to interpret their images, but for the clinician it is a prerequisite to be able to immediately interpret the dynamic images and correlate the ultrasound findings with the patient's symptoms and signs. This emphasizes the need for future studies to investigate whether placing ultrasound devices in the hands of medical students will improve medical practice at the point of care.

\section{Conclusion}

$\nabla$

Medical students with no previous experience of ultrasound techniques demonstrated a significant increase in their ability to acquire and interpret an ultrasound image after completion of interactive e-learning, and this competence was further improved after $4 \mathrm{~h}$ of systematic hands-on training. Such training should translate into improved medical care in qualified doctors.

\section{Affiliations}

' Department of Cardiothoracic \& Vascular Surgery, Aarhus University Hospital, Aarhus, Denmark

${ }^{2}$ Department of Anaesthesia and Intensive Care, Aarhus University Hospital, Aarhus, Denmark

${ }^{3}$ Department of Anaesthesia, Groote Schuur Hospital, University of Cape

Town, Cape Town, South Africa

\section{References}

1 Moore CL, Copel JA. Point-of-care ultrasonography. The New England Journal of Medicine 2011; 364: 749-757

2 Labovitz AJ, Noble VE, Bierig $M$ et al. Focused cardiac ultrasound in the emergent setting: a consensus statement of the American Society of Echocardiography and American College of Emergency Physicians. Journal of the American Society of Echocardiography 2010; 23: $1225-1230$

3 Spencer KT, Kimura BJ, Korcarz CE et al. Focused cardiac ultrasound: recommendations from the American Society of Echocardiography. Journal of the American Society of Echocardiography 2013; 26: 567-581

4 Neskovic AN, Hagendorff A, Lancellotti P et al. Emergency echocardiography: the European Association of Cardiovascular Imaging recommendations. European Heart Journal Cardiovascular Imaging 2013; 14: $1-11$

5 Neskovic AN, Edvardsen T, Galderisi $M$ et al. Focus cardiac ultrasound: the European Association of Cardiovascular Imaging viewpoint. European Heart Journal Cardiovascular Imaging 2014;

6 Laursen $C B$, Sloth E, Lambrechtsen J et al. Focused sonography of the heart, lungs, and deep veins identifies missed life-threatening conditions in admitted patients with acute respiratory symptoms. Chest 2013; 144: 1868-1875

7 Laursen CB, Sloth E, Lassen AT et al. Point-of-care ultrasonography in patients admitted with respiratory symptoms: a single-blind, randomised controlled trial. The lancet Respiratory Medicine 2014

8 Melniker LA, Leibner E, McKenney MG et al. Randomized controlled clinical trial of point-of-care, limited ultrasonography for trauma in the emergency department: the first sonography outcomes assessment program trial. Annals of Emergency Medicine 2006; 48: 227-235

9 Hansen MA, Juhl-Olsen P, Thorn S et al. Ultrasonography-guided radial artery catheterization is superior compared with the traditional palpation technique: a prospective, randomized, blinded, crossover study. Acta Anaesthesiologica Scandinavica 2014; 58: 446-452

10 Verghese ST, McGill WA, Patel RI et al. Comparison of three techniques for internal jugular vein cannulation in infants. Paediatric Anaesthesia 2000; 10: 505-511

11 Neri L, Storti E, Lichtenstein D. Toward an ultrasound curriculum for critical care medicine. Critical Care Medicine 2007; 35 (5 Suppl): 290-304

12 Randolph AG, Cook DJ, Gonzales CA et al. Ultrasound guidance for placement of central venous catheters: a meta-analysis of the literature. Critical Care Medicine 1996; 24: 2053-2058

13 Solomon $S D$, Saldana F. Point-of-care ultrasound in medical education - stop listening and look. The New England Journal of Medicine 2014; 370: 1083-1085

14 Hoppmann $R A$, Rao $V V$, Poston $M B$ et al. An integrated ultrasound curriculum (iUSC) for medical students: 4-year experience. Critical Ultrasound Journal 2011; 3: 1-12

15 Bahner DP, Royall NA. Advanced ultrasound training for fourth-year medical students: a novel training program at The Ohio State University College of Medicine. Academic Medicine 2013; 88: 206-213

16 Rao S, van Holsbeeck L, Musial JL et al. A pilot study of comprehensive ultrasound education at the Wayne State University School of Medicine: a pioneer year review. Journal of Ultrasound in Medicine 2008; 27: 745-749

17 Brunner $M$, Moeslinger T, Spieckermann PG. Echocardiography for teaching cardiac physiology in practical student courses. The American Journal of Physiology 1995; 268: 2-9

18 Teichgraber UK, Meyer JM, Poulsen Nautrup C et al. Ultrasound anatomy: a practical teaching system in human gross anatomy. Medical Education 1996; 30: 296-298 
19 Wicke W, Brugger PC, Firbas W. Teaching ultrasound of the abdomen and the pelvic organs in the medicine curriculum in Vienna. Medical Education 2003; 37: 476

20 Lamperti $M$, Bodenham AR, Pittiruti $M$ et al. International evidencebased recommendations on ultrasound-guided vascular access. Intensive Care Medicine 2012; 38: 1105-1117

21 Via G, Hussain A, Wells $M$ et al. International evidence-based recommendations for focused cardiac ultrasound. Journal of the American Society of Echocardiography 2014; 27: 683.e1-683.e33

22 Volpicelli G, Elbarbary M, Blaivas $M$ et al. International evidence-based recommendations for point-of-care lung ultrasound. Intensive Care Medicine 2012; 38: 577-591

23 Jensen $M B$, Sloth E, Larsen KM et al. Transthoracic echocardiography for cardiopulmonary monitoring in intensive care. European Journal of Anaesthesiology 2004; 21: 700-707

24 Scalea TM, Rodriguez A, Chiu WC et al. Focused Assessment with Sonography for Trauma (FAST): results from an international consensus conference. The Journal of Trauma 1999; 46: 466-472

25 Tso P, Rodriguez A, Cooper C et al. Sonography in blunt abdominal trauma: a preliminary progress report. The Journal of Trauma 1992; 33: $39-43$
26 Clemmesen L, Knudsen L, Sloth E et al. Dynamic needle tip positioning - ultrasound guidance for peripheral vascular access. A randomized, controlled and blinded study in phantoms performed by ultrasound novices. Ultraschall in der Medizin 2012; 33: 321-325

27 Jakobsen CJ, Torp P, Sloth E. Perioperative feasibility of imaging the heart and pleura in patients with aortic stenosis undergoing aortic valve replacement. European Journal of Anaesthesiology 2007; 24: 589-595

28 Fernandez-Frackelton $M$, Peterson $M$, Lewis RJ et al. A bedside ultrasound curriculum for medical students: prospective evaluation of skill acquisition. Teaching and Learning in Medicine 2007; 19: 14-19

29 Gogalniceanu P, Sheena $Y$, Kashef E et al. Is basic emergency ultrasound training feasible as part of standard undergraduate medical education? Journal of Surgical Education 2010; 67: 152-156

30 Breitkreutz R, Price S, Steiger HV et al. Focused echocardiographic evaluation in life support and peri-resuscitation of emergency patients: a prospective trial. Resuscitation 2010; 81: 1527-1533 


\section{Appendix}

$\nabla$

\section{a Cardiac and pleural ultrasound}

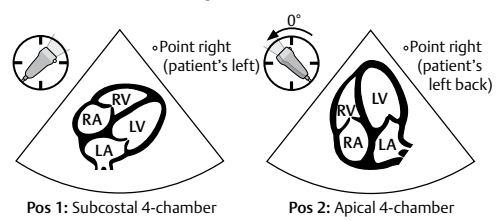

(3) $\begin{gathered}\text { Ppoint left } \\ \text { (patient's rigg }\end{gathered}$

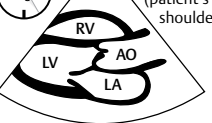

Pos 3: Parasternal long axis

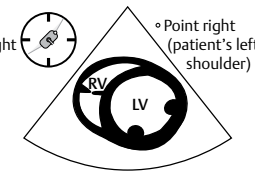

Pos 3: Parasternal LV short axis
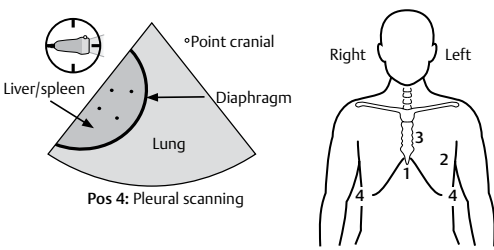
Lung ultrasound (LUS)
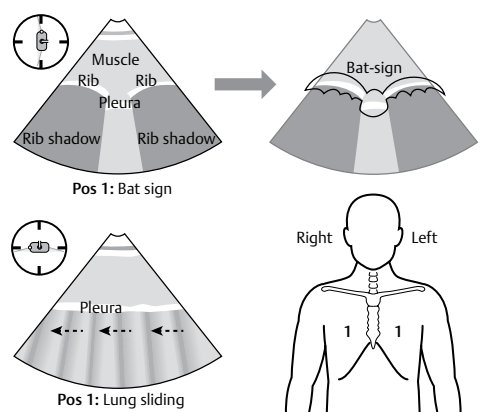

b

Abdominal ultrasound

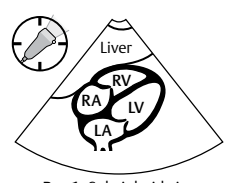

Pos 1: Subxiphoid view

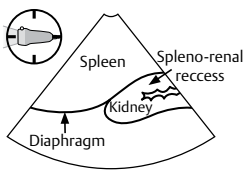

Pos 3: Left upper quadrant
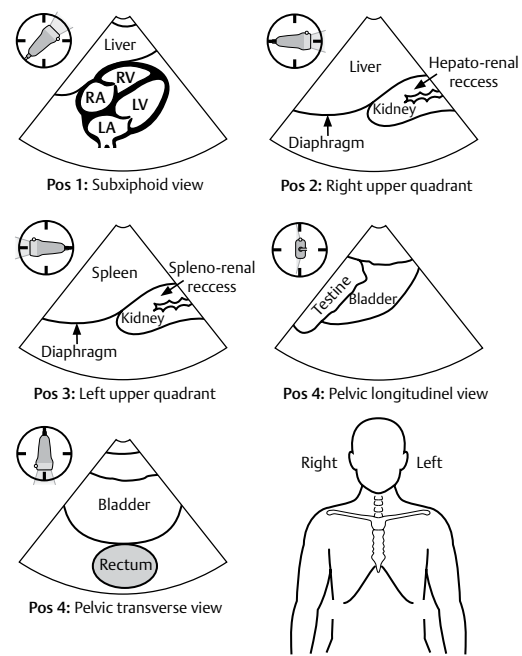

d

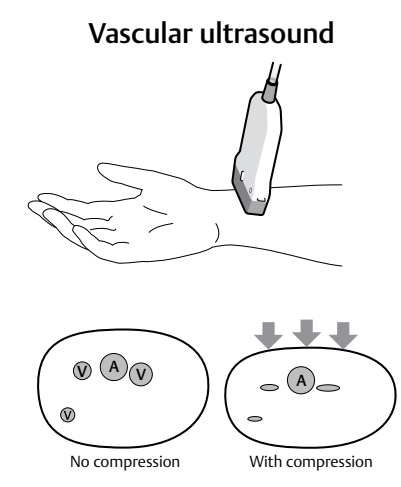

Appendix 1 Posters. Posters presented to students during test sessions. a Cardiac and pleural ultrasound; b Abdominal ultrasound; c Lung ultrasound; $\mathbf{d}$ Vascular ultrasound. 